\title{
GROUP ALGEBRAS WITH NILPOTENT UNIT GROUPS
}

\author{
J. M. BATEMAN AND D. B. COLEMAN
}

In this note we determine under what conditions the group of units in the group algebra of a finite group is nilpotent.

Let $G$ be a finite group, let $F$ be a field of characteristic $p$, and let $F(G)$ denote the group algebra of $G$ over $F$. The group of units in $F(G)$ will be denoted by $U=U(G, F)$.

TheOREM. (a) Let $F(G)$ be semisimple. Then $U$ is nilpotent if and only if $G$ is abelian.

(b) Let $p$ be a prime dividing the order of $G$. Then $U$ is nilpotent if and only if $G$ is nilpotent with abelian $q$-Sylow subgroup for each prime $q \neq p$.

Proof. (a) Suppose that $G$ is nonabelian. Since $F(G)$ is semisimple, we have

$$
F(G) \cong M_{n_{1}}\left(D_{1}\right) \oplus \cdots \oplus M_{n_{8}}\left(D_{8}\right),
$$

where each $D_{i}$ is a division algebra over $F ; M_{n_{i}}\left(D_{i}\right)$ denotes the total matric algebra over $D_{i}$.

$U(G, F)$ is thus the direct product of the general linear groups $\mathrm{GL}\left(n_{i}, D_{i}\right), i=1, \cdots, s$.

Suppose that $p \neq 0$. If $n_{i}=1$, then the division algebra $D_{i}$ is spanned by a homomorphic image of $G$; hence by [2], $D_{i}$ is a field. Thus some $n_{j}$ exceeds 1 . For such an $n_{j}, \mathrm{GL}\left(n_{j}, D_{j}\right)$ is not nilpotent; hence $U$ is not nilpotent.

Suppose that $p=0$. If some $n_{i}$ is greater than 1 , then as above, $U$ is not nilpotent. Assume that each $n_{i}=1$. Then $F(G)$ is the direct sum of division algebras, so according to [1], $G$ is Hamiltonian and $U$ has the group of nonzero rational quaternions as a subgroup. It is easy to see that this group is not nilpotent; hence neither is $U$.

(b) The following easy result gives a convenient way of handling the sufficiency part of (b) when $F$ is infinite.

Lemma. Let $R$ be a ring with identity. Suppose that $R$ has a nilpotent ideal $N$ such that $R / N$ is commutative. Then the group $V$ of units in $R$ is nilpotent.

Proof. For $x$ and $y$ in $R$, we have by hypothesis that the Lie product $[x, y]=x y-y x$ is in $N$.

Received by the editors January 12, 1967. 
For $u$ and $v$ in $V$, define $(u, v)=u^{-1} v^{-1} u v$; for elements $u_{1}, \cdots, u_{n}$ in $V$, define inductively $\left(u_{1}, \cdots, u_{n}\right)=\left(\left(u_{1}, \cdots, u_{n-1}\right), u_{n}\right)$. Using the equations

$$
\left(u_{1}, \cdots, u_{n}\right)=1+\left(u_{1}, \cdots, u_{n-1}\right)^{-1} u_{n}^{-1}\left[\left(u_{1}, \cdots, u_{n-1}\right), u_{n}\right]
$$

and

$$
[x-1, y]=[x, y] \text {, }
$$

we see by induction that for $n>1$ and for $u_{1}, \cdots, u_{n}$ in $V$, $\left(u_{1}, \cdots, u_{n}\right)-1$ is in $N^{n-1}$. Since $N$ is a nilpotent ideal, we have that $V$ is a nilpotent group. This proves the lemma.

If $U$ is nilpotent, then so is $G$. Assume this to be the case and let $A$ be the $p$-complement of $G$. Then $U(A, F)$ is a subgroup of $U$ and hence is nilpotent. By part (a), $A$ is abelian.

Conversely, let $G=P \times A$, where $P$ is a $p$-group (perhaps trivial) and $A$ is abelian of order prime to $p$. Suppose that $F(A)$ $=F_{1} \oplus \cdots \oplus F_{k}$, where each $F_{i}$ is a field over $F$. Then

$$
F(G) \cong F_{1} \otimes F(P) \oplus \cdots \oplus F_{k} \otimes F(P) .
$$

As a ring, each $F_{i} \otimes F(P) \cong F_{i}(P)$. Since $P$ is a p-group, $F(G)$ modulo its radical is isomorphic to $F(A)$. Thus by the lemma, $U$ is nilpotent.

\section{REFERENCES}

1. S. D. Berman, On certain properties of integral group rings, Dokl. Akad. Nauk SSSR 91 (1953), 7-9. (Russian)

2. I. N. Herstein, Finite multiplicative subgroups in division rings, Pacific J. Math. 3 (1953), 121-126.

UNIVERSITY OF KENTUCKY 